\title{
MEG Assessment of Expressive Language in Children Evaluated for Epilepsy Surgery
}

\author{
Elaine Foley ${ }^{1} \cdot$ J. Helen Cross ${ }^{3,4} \cdot$ Ngoc J. Thai $^{5} \cdot$ A. Richard Walsh ${ }^{2} \cdot$ Peter Bill ${ }^{2} \cdot$ Paul Furlong $^{1} \cdot$ Amanda G. Wood $^{1,7}$. \\ Antonella Cerquiglini ${ }^{6} \cdot$ Stefano Seri ${ }^{1,2} \mathbb{0}$
}

Received: 18 October 2018 / Accepted: 7 March 2019 / Published online: 20 March 2019

(c) The Author(s) 2019

\begin{abstract}
Establishing language dominance is an important step in the presurgical evaluation of patients with refractory epilepsy. In the absence of a universally accepted gold-standard non-invasive method to determine language dominance in the preoperative assessment, a range of tools and methodologies have recently received attention. When applied to pediatric age, many of the proposed methods, such as functional magnetic resonance imaging (fMRI), may present some challenges due to the time-varying effects of epileptogenic lesions and of on-going seizures on maturational phenomena. Magnetoencephalography (MEG) has the advantage of being insensitive to the distortive effects of anatomical lesions on brain microvasculature and to differences in the metabolism or vascularization of the developing brain and also provides a less intimidating recording environment for younger children. In this study we investigated the reliability of lateralized synchronous cortical activation during a verb generation task in a group of 28 children ( 10 males and 18 females, mean age 12 years) with refractory epilepsy who were evaluated for epilepsy surgery. The verb generation task was associated with significant decreases in beta oscillatory power $(13-30 \mathrm{~Hz})$ in frontal and temporal lobes. The MEG data were compared with other available presurgical non-invasive data including cortical stimulation, neuropsychological and fMRI data on language lateralization where available. We found that the lateralization of MEG beta power reduction was concordant with language dominance determined by one or more different assessment methods (i.e. cortical stimulation mapping, neuropsychological, fMRI or post-operative data) in $89 \%$ of patients. Our data suggest that qualitative hemispheric differences in task-related changes of spectral power could offer a promising insight into the contribution of dominant and non-dominant hemispheres in language processing and may help to characterize the specialization and lateralization of language processes in children.
\end{abstract}

Keywords Children $\cdot$ Magnetoencephalography $\cdot$ Language lateralization $\cdot$ Hemispheric dominance $\cdot$ Beamformers . Functional mapping $\cdot$ Epilepsy surgery

\section{Introduction}

Identification of language-relevant cortex in pediatric age is one of the most challenging aspects of the pre-surgical evaluation process for frontal and temporal lobe epilepsies,

Handling Editor: Christoph M. Michel.

Stefano Seri

s.seri@aston.ac.uk

1 Aston Brain Centre, School of Life and Health Sciences, Aston University, Birmingham, UK

2 Children's Epilepsy Surgery Service, Birmingham Children's and Women's Hospital NHS Foundation Trust, Birmingham, UK

3 Developmental Neurosciences Programme, Institute of Child Health, University College London, London, UK
4 Department of Paediatric Neurology, Great Ormond Street Hospital NHS Foundation Trust, London, UK

5 CRICBristol, Bristol's Clinical Research and Imaging Centre, Bristol, UK

6 Developmental Neuropsychiatry Section, Dipartimento di Scienze e BiotecnologieMedico-Chirurgiche, "Sapienza" Universita' di Roma Polo Pontino, Latina, Italy

7 Brain and Mind, Clinical Sciences, Murdoch Children's Research Institute, Melbourne, Australia 
further complicated by the high incidence of atypical language organization in patients with a history of drug-resistant epilepsy (Duchowny et al. 1996; Hamberger and Cole 2011). A range of non-invasive tools and methodologies to assess language dominance have been developed with the ultimate aim of replacing the Intracarotid Amobarbital Procedure (IAP), a procedure accepted for decades as the goldstandard diagnostic test in adults (Abou-Khalil 2007). When applied to pediatric age, the reliability of the proposed methods is influenced among others by the time-varying effects of the epileptogenic lesion and of the on-going seizures on maturational phenomena (Holmes 2016). Furthermore, the tolerability of currently available diagnostic procedures can make the assessment of hemispheric dominance for language and interpretation in children particularly challenging (de Ribaupierre et al. 2012).

Functional MRI is the most widely used of the minimally/ non-invasive techniques for presurgical language mapping in children (Rodin et al. 2013), but MEG is gaining increasing acceptance (Pang et al. 2011). Compared to MRI, methods based on electromagnetic measures of brain activity have the advantage of being insensitive to the distortive effects of anatomical lesions on brain microvasculature or metabolism on the developing brain (Demonet et al. 2005) and also provide a less intimidating recording environment for younger children (Pang et al. 2011). Initial reports on MEG language localization have shown encouraging results with a wide variety of cognitive paradigms (Pirmoradi et al. 2010) and analysis strategies (Edwards et al. 2010; Hirata et al. 2010; McDonald et al. 2010; Papanicolaou et al. 2004). The suitability of MEG language mapping protocols in adults as an alternative to the Wada procedure have been addressed with validation studies conducted with various approaches and methodologies and overall high concordance rates (Bowyer et al. 2005; Findlay et al. 2012; Hirata et al. 2004; Maestu et al. 2002; Merrifield et al. 2007; Papanicolaou et al. 2004; Tanaka et al. 2013).

Adult MEG studies using equivalent current dipole models or distributed source models to localize evoked activity have reported good concordance (75-90\%) with findings from the IAP (Breier et al. 2001; Doss et al. 2009; McDonald et al. 2010; Papanicolaou et al. 2004). More recently, task-related time-varying changes in the electromagnetic power spectrum have been used to detect hemispheric dominance during language tasks and have also demonstrated high concordance with IAP ranging from 85 to $95 \%$ (Findlay et al. 2012; Hirata et al. 2004; Kim and Chung 2008). A study of the relationship between MEG localization of language-sensitive areas and electrical stimulation mapping in two adults (Simos et al. 1999a) added convergent evidence that MEG is a useful, non-invasive method for assessing hemispheric language dominance in adults with refractory seizures who are surgical candidates. Yet despite the overall positive contribution of MEG in the assessment of language localization in typically developing individuals, data on its clinical use in clinical pediatric populations is still relatively modest. Recent studies have reported the feasibility of passive paradigms in children and adolescents under sedation (Rezaie et al. 2014) and during sleep (Van Poppel et al. 2012), but MEG studies using more traditional expressive language tasks in pre-surgical assessment of children are still limited.

The aim of this study was to provide a contribution to addressing this gap by measuring hemispheric lateralization of MEG oscillatory activity during awake expressive language processing in pediatric age in a naturalistic presurgical setting. We investigated feasibility and reliability of pre-operative MEG assessment of language lateralization in 28 pediatric patients using a verb generation task.

\section{Methods}

\section{Participants}

Twenty-eight pediatric patients with drug-resistant epilepsy under evaluation for resective surgery participated in the study. Ten male and eighteen females (mean age 12 years; age range 7-18 years) were included in the study (see Table 1 for a summary of patient demographics). All patients were referred to the Wellcome Laboratory for MEG studies at the Aston Brain Centre in Birmingham from the two largest centers participating in the Children's Epilepsy Surgery Service (CESS) program of NHS England between 2013 and 2016 for localization of the irritative zone and eloquent cortex mapping. Twenty patients were referred from Birmingham Women's and Children's Hospital NHS Foundation Trust and eight from Great Ormond Street Hospital for Children NHS Foundation Trust. Inclusion criteria included being native English speakers and the ability to perform the task during a practice session prior to the MEG recording. Informed consent was obtained from all patients prior to MEG recording and it was established from seizure diary sheets that all patients were seizure-free for at least $24 \mathrm{~h}$ prior to recording. The study was conducted according to the ethical principles of the declaration of Helsinki and approved by the UK NHS research ethics committee (Ref. 17/WM/0174).

\section{Experimental Paradigm}

We used a 'child-friendly' verb generation task described in a previous study by our group (Fisher et al. 2008). In this task participants were visually presented with a series of single nouns and asked to generate an associated verb for each (e.g. 'BALL'-'throw'/'catch'). To separate out component 
Table 1 Summary of patient characteristics and results

\begin{tabular}{|c|c|c|c|c|c|c|c|c|c|}
\hline Pt. & $\begin{array}{l}\text { Age } \\
\text { (years), } \\
\text { Gender }\end{array}$ & $\begin{array}{l}\text { Age at } \\
\text { Onset } \\
\text { (y) }\end{array}$ & Handedness & Etiology & $\begin{array}{l}\text { Seizure } \\
\text { lateraliza- } \\
\text { tion }\end{array}$ & Surgical procedure & $\begin{array}{l}\text { Language laterali- } \\
\text { zation: MEG }\end{array}$ & $\begin{array}{l}\text { Language laterali- } \\
\text { zation: neuropsy- } \\
\text { chology and fMRI }\end{array}$ & $\begin{array}{l}\text { Language } \\
\text { lateralization: } \\
\text { DCS }\end{array}$ \\
\hline 1 & $7, \mathrm{M}$ & 2 & $\mathrm{R}$ & TSC & $\mathrm{L}$ & L ATL & $\mathrm{LI}=29.35, \mathrm{~L}$ & $\mathrm{~L}$ & $\mathrm{~L}$ \\
\hline 2 & $8, \mathrm{M}$ & 5 & $\mathrm{R}$ & FCD & $\mathrm{L}$ & L ATL+ & $\mathrm{LI}=19.82, \mathrm{~L}$ & $\mathrm{~L}$ & $\mathrm{~L}$ \\
\hline 3 & $9, \mathrm{~F}$ & 1 & $\mathrm{R}$ & MTS & $\mathrm{L}$ & L ATL+ & $\mathrm{LI}=21.39, \mathrm{~L}$ & $\mathrm{~L}$ & $\mathrm{~L}$ \\
\hline 4 & $7, \mathrm{~F}$ & 5 & $\mathrm{R}$ & FCD & $\mathrm{L}$ & L ATL & $\mathrm{LI}=60.35, \mathrm{~L}$ & $\mathrm{~L}$ & $\mathrm{~L}$ \\
\hline 5 & $8, \mathrm{~F}$ & 3 & $\mathrm{R}$ & MTS & $\mathrm{L}$ & L ATL+ & $\mathrm{LI}=26.03, \mathrm{~L}$ & $\mathrm{~L}$ & $\mathrm{~L}$ \\
\hline 6 & $8, \mathrm{M}$ & 1 & $\mathrm{R}$ & FCD & $\mathrm{L}$ & L ATL+ & $\mathrm{LI}=33.02, \mathrm{~L}$ & $\mathrm{~L}$ & $\mathrm{~L}$ \\
\hline 7 & $11, \mathrm{~F}$ & 1 & $\mathrm{R}$ & MTS & $\mathrm{L}$ & L ATL+ & $\mathrm{LI}=58.62, \mathrm{~L}$ & $\mathrm{~L}$ & $\mathrm{~L}$ \\
\hline 8 & $10, \mathrm{~F}$ & 1 & $\mathrm{R}$ & MTS & $\mathrm{L}$ & L ATL+ & $\mathrm{LI}=28.09, \mathrm{~L}$ & $\mathrm{~L}$ & $\mathrm{~L}$ \\
\hline 9 & $14, \mathrm{M}$ & 2 & $\mathrm{R}$ & Ganglioma & $\mathrm{R}$ & $\begin{array}{l}\text { R Temporal } \\
\text { Lesionectomy }\end{array}$ & $\mathrm{LI}=76.31, \mathrm{~L}$ & $\mathrm{~L}$ & 1 \\
\hline 10 & $16, F$ & 2 & $\mathrm{R}$ & FCD & $\mathrm{L}$ & L ATL+ & $\mathrm{LI}=52.43, \mathrm{~L}$ & $\mathrm{~L}$ & 1 \\
\hline 11 & $17, \mathrm{~F}$ & 1 & $\mathrm{~L}$ & Stroke & $\mathrm{L}$ & L Hemispherotomy & $\mathrm{LI}=-47.91, \mathrm{R}$ & $\mathrm{R}$ & 1 \\
\hline 12 & $17, \mathrm{~F}$ & 10 & $\mathrm{R}$ & FCD & $\mathrm{R}$ & R ATL & $\mathrm{LI}=38.66, \mathrm{~L}$ & $\mathrm{~L}$ & 1 \\
\hline 13 & $16, F$ & 10 & $\mathrm{R}$ & FCD & $\mathrm{L}$ & L ATL & $\mathrm{LI}=64.95, \mathrm{~L}$ & $\mathrm{~L}$ & $\mathrm{~L}$ \\
\hline 14 & $16, F$ & 13 & $\mathrm{~L}$ & FCD & $\mathrm{L}$ & L ATL+ & $\mathrm{LI}=-80.62, \mathrm{R}$ & $\mathrm{L}$ & 1 \\
\hline 15 & $15, \mathrm{~F}$ & 2 & $\mathrm{~L}$ & FCD & $\mathrm{L}$ & No Surgery & $\mathrm{LI}=4.77$, Bilat & Bilat & 1 \\
\hline 16 & $8, \mathrm{M}$ & 6 & $\mathrm{~L}$ & FCD & $\mathrm{L}$ & L Hemispherotomy & $\mathrm{LI}=-51.26, \mathrm{R}$ & $\mathrm{R}$ & 1 \\
\hline 17 & $15, \mathrm{~F}$ & 3 & $\mathrm{R}$ & TSC & $\mathrm{L}$ & $\begin{array}{l}\text { R Frontal } \\
\text { Lesionectomy }\end{array}$ & $\mathrm{LI}=-34.93, \mathrm{R}$ & $\mathrm{L}$ & 1 \\
\hline 18 & $12, \mathrm{M}$ & 1 & L/Ambi & MTS & $\mathrm{L}$ & L ATL & $\mathrm{LI}=-13.46$, Bilat & Bilat & 1 \\
\hline 19 & $18, \mathrm{M}$ & 4 & $\mathrm{R}$ & MRI Neg & $\mathrm{R}$ & No Surgery & $\mathrm{LI}=36.51, \mathrm{~L}$ & $\mathrm{~L}$ & 1 \\
\hline 20 & $11, \mathrm{M}$ & 8 & $\mathrm{~L}$ & FCD II & $\mathrm{L}$ & L ATL & $\mathrm{LI}=98.02, \mathrm{~L}$ & $\mathrm{~L}$ & 1 \\
\hline 21 & $14, \mathrm{~F}$ & 1 & $\mathrm{R} / \mathrm{Ambi}$ & FCD & $\mathrm{L}$ & No Surgery & $\mathrm{LI}=61.77, \mathrm{~L}$ & $\mathrm{~L}$ & 1 \\
\hline 22 & $16, \mathrm{M}$ & 10 & $\mathrm{R}$ & FCD Ib & $\mathrm{R}$ & $\begin{array}{l}\text { R Frontal } \\
\text { Lesionectomy }\end{array}$ & $\mathrm{LI}=63.68, \mathrm{~L}$ & $\mathrm{~L}$ & $\mathrm{~L}$ \\
\hline 23 & $17, \mathrm{~F}$ & 7 & $\mathrm{R}$ & MRI Neg & $\mathrm{L}$ & No Surgery & $\mathrm{LI}=33.81, \mathrm{~L}$ & $\mathrm{~L}$ & 1 \\
\hline 24 & $11, \mathrm{M}$ & 2 & $\mathrm{R}$ & FCD & $\mathrm{R}$ & No Surgery & $\mathrm{LI}=86.17, \mathrm{~L}$ & $\mathrm{~L}$ & 1 \\
\hline 25 & $10, \mathrm{~F}$ & 5 & $\mathrm{R}$ & MRI Neg & $\mathrm{L}$ & No Surgery & $\mathrm{LI}=86.83, \mathrm{~L}$ & Bilat & 1 \\
\hline 26 & $12, \mathrm{~F}$ & 2 & $\mathrm{R}$ & MRI Neg & $\mathrm{R}$ & $\begin{array}{l}\text { R Temporal Resec- } \\
\text { tion }\end{array}$ & $\mathrm{LI}=42.78, \mathrm{~L}$ & $\mathrm{~L}$ & 1 \\
\hline 27 & $16, \mathrm{~F}$ & 11 & $\mathrm{R}$ & MRI Neg & $\mathrm{L}$ & No Surgery & $\mathrm{LI}=81.76, \mathrm{~L}$ & $\mathrm{~L}$ & 1 \\
\hline 28 & $15, \mathrm{~F}$ & 5 & $\mathrm{R}$ & FCD Ia & $\mathrm{R}$ & $\begin{array}{l}\text { R Frontal \& Pari- } \\
\text { etal Resection }\end{array}$ & $\mathrm{LI}=30.26, \mathrm{~L}$ & $\mathrm{~L}$ & 1 \\
\hline
\end{tabular}

$L$ left, $R$ right, $A m b i$ ambidextrous, Bilat bilateral, $A T L$ anterior temporal lobectomy, $A T L+$ anterior temporal lobectomy + hippocampoamygdalectomy, TSC Tuberous Sclerosis Complex, FCD focal cortical dysplasia, MTS mesial temporal sclerosis, MRI Neg MRI negative, LI lateralization index

processes involved in articulation and associated muscle artefacts, participants were instructed to initially generate responses covertly and then to vocalize their response on presentation of a visual cue. The overt component of the task was included to determine that participants were performing the task correctly. The task commenced with a three-second 'passive' phase where participants were asked to focus on a fixation cross. Then a noun was visually presented and participants were instructed to silently generate their response (three second 'active' phase). This was followed by an image of Mr. Chatterbox (Copyright (c) 2018 THOIP - a Sanrio company) cueing participants to verbalize their response.
The Mr. Chatterbox $@$ image remained on the screen for $3 \mathrm{~s}$ followed by an inter-stimulus interval of $500 \mathrm{~ms}$ before the onset of the next trial. Sixty data trials were collected for this task. Visual stimuli were presented using Presentation software (Neurobehavioral Systems, Inc.). Fixation was monitored with a camera system positioned in front of the patient with on-line tracking of eye movement. Epochs with changes in fixation point greater than $1 \mathrm{~cm}$ were discarded from the analysis. The stimuli were projected using a projector located outside of the shielded room and projected on to a translucent screen, which was positioned $85 \mathrm{~cm}$ from the participant subtending a 3-degree visual angle. 
High-resolution anatomical MRI scans (3D inversion recovery whole-head volume sequences were acquired with $1 \mathrm{~mm}^{3}$ isotropic resolution) previously acquired within a 3-month interval at the two referring centers as part of the presurgical evaluation, were used for co-registration with the MEG data.

\section{MEG Data Acquisition}

MEG data were recorded in a magnetically shielded room using an Elekta-Neuromag TRIUX whole-head system (Helsinki, Finland) with 204 planar dc-SQUID gradiometers and 102 magnetometers. Participants were seated in an upright position in the MEG scanner. Data were acquired with 2 $\mathrm{KHz}$ sampling rate, and low-pass filter of $660 \mathrm{~Hz}$. One bipolar EEG channel was dedicated to recording ECG. Five coils were placed on the patient's head, three on the front and one on each mastoid for continuous monitoring of head position. To allow the translation between the MEG coordinate system and the patient's structural MRI, three head position fiducial points at the nasion, left and right pre-auricular points were digitized with a Polhemus Fastrack device, which was also used to measure the surface shape of each participant's head and to localize the electromagnetic head coils with respect to that surface. Each participant's head shape file was then extracted and co-registered with the high-resolution anatomical MRI sequence. Co-registration was performed using inhouse software based on an algorithm designed to minimize the squared Euclidean distance between the Polhemus and the MRI surfaces. The accuracy of this procedure has been shown to be within $5 \mathrm{~mm}$ (Adjamian et al. 2004).

\section{MEG Data Analysis}

Artefacts were removed from the raw data with MaxFilter software (Elekta Neuromag Oy, version 2.2.10) that uses the temporal extension of signal space separation (tSSS) (Taulu and Hari 2009). Max-filtered data were visually inspected to identify "bad channels" that were removed if present. The data were then analyzed in the Matlab R2012a environment (The MathWorks Inc., Natick, MA.) using the FieldTrip toolbox (Oostenveld et al. 2011). Responses to the overt segment of each trial were initially inspected for accuracy and for missed responses. Data analysis was then performed on the correct covert trials only; $5 \mathrm{~s}$ epochs were created based on $2.5 \mathrm{~s}$ pre- and $2.5 \mathrm{~s}$ post-stimulus. Data epochs of interest were then visually inspected for any additional artefacts caused by muscle activity or SQUID jumps and any contaminated trials were discarded. Time windows with relatively long duration were chosen to capitalize on the sustained nature of task-induced spectral changes and differentiate these effects from transient responses related to stimulus delivery.

\section{Whole Brain Analysis}

Individual head shapes were created for each patient based on their own structural MRI and were co-registered to the MEG coordinate system. Realistic, single-shell brain models were also constructed for each participant based on their structural MRIs (Nolte 2003). An MEG sourcespace voxel size of $0.5 \mathrm{~cm}$ was used to compute the lead field matrix. An adaptive spatial filtering beamforming technique in the frequency-domain, known as dynamic imaging of coherent sources (DICS), was used to determine sources of neuronal activity associated with the verb generation task (Gross et al. 2001). Spectral perturbation data in the source space were produced for the beta frequency range $(13-30 \mathrm{~Hz})$. The choice of frequency-band was selected based on previous evidence demonstrating spectral power changes within this range during language processing (Findlay et al. 2012; Fisher et al. 2008; Hirata et al. 2010; Pang et al. 2011). At the individual level, statistical differences between the active and baseline conditions were calculated using a paired-sample $t$ test. A nonparametric randomization test was then used to correct for multiple comparisons (Maris and Oostenveld 2007). Source estimates were interpolated onto the individual anatomical images and results are displayed on individual patient's T1 MRIs. Source estimates were subsequently normalized to a standard MNI template brain for illustrative purposes only.

Lateralization index (LI) was computed based on t-values of the event related power decrease in Brodmann areas (BA) 6, 44, 45 and 22 in the left $\left(\mathrm{t}_{\mathrm{L}}\right)$ and right hemisphere $\left(t_{\mathrm{R}}\right)$. These regions of interest were selected based on the volumes of interest used in previous clinical MEG studies of language (Findlay et al. 2012; Hirata et al. 2004) and they represent areas that are commonly explored with direct cortical stimulation, including Broca (BA44/45) and Wernicke's (BA22) areas (Ojemann et al. 2003). The $\mathrm{t}$-values of the most prominent decrease in oscillatory power within the $13-30 \mathrm{~Hz}$ frequency range within the related region and its contralateral homologous region were selected.

The index used was:

$L I=100 * \frac{\left(t_{L}-t_{R}\right)}{\left(\left|t_{L}\right|+\left|t_{R}\right|\right)}$

A positive value indicated greater power decrease in left hemisphere and a negative value in the right. The use of lateralization indices in neuroimaging studies has received some criticism as they are sensitive to user defined thresholds (Chlebus et al. 2007; Jansen et al. 2006; Rutten et al. 2002) and rely upon decisions about the choice of total 
or peak strength within regions of interest (Fisher et al. 2008). However, the lateralization index can be a useful measure to address the problem of excessive false positives in language studies where bilateral activation is common (Findlay et al. 2012; Papanicolaou et al. 2004). Here it was used as an additional quantitative measure to complement the qualitative assessment of spectral power analysis.

\section{Regions of Interest Analysis}

The output of the beamformer has been referred to as 'virtual electrode' or 'virtual sensor' which can be visualized as time-frequency representations of activity arising from specific voxels where spectral power changes are identified (Singh et al. 2003) and used to characterize in greater detail frequency-specific spectral power changes associated with the task (Hillebrand et al. 2005). Virtual electrodes were constructed based on the data covariance matrix using a $5 \mathrm{~s}$ window from $2.5 \mathrm{~s}$ pre-stimulus to $2.5 \mathrm{~s}$ post stimulus onset, with a bandwidth of $1-30 \mathrm{~Hz}$. The difference in source estimates between pre-stimulus baseline and task-related activity was evaluated, in specific regions of interest corresponding to Brodmann areas 6, 44, 45 and 22.

Time-frequency analysis on the virtual sensor signal was performed using EEGLab software version 11.0.0.0b (Delorme and Makeig 2004) running in Matlab R2012a. Single-trial epochs were analyzed using a moving window short-time Fourier transform with 200 overlapping time windows per trial. The average log-power in the baseline period was subtracted from the log-power at each time-frequency point in the active period, yielding the measure conventionally known as "event-related spectral perturbation," or ERSP (Makeig 1993). The color at each image pixel in the spectrogram then indicates power (in $\mathrm{dB}$ ) at a given frequency and latency relative to the stimulus. Significance of deviations from baseline power was assessed using a bootstrap method, where a surrogate data distribution was constructed by selecting spectral estimates for each trial from randomly selected latency windows in the baseline period and then averaging them. This process was applied 200 times to produce a surrogate 'baseline' amplitude distribution whose specified percentiles were then used as significance thresholds, with $\mathrm{p}<.05$ after correcting for multiple comparisons using the FDR method.

\section{Comparison Modalities}

\section{Neuropsychological Testing and fMRI}

Full neuropsychological testing was performed on all patients as part of their routine surgical workup and included measures of handedness, language competence and IQ. These were assessed according to standardized administration of age-appropriate measures. Intellectual ability was assessed with the Wechsler Abbreviated Scale of Intelligence (WASI, Elliott 1990; Wechsler 1999) which includes measures of verbal and non-verbal abilities and gives rise to a measure of full scale IQ (FSIQ). Verbal fluency was assessed using the Delis-Kaplan Executive Function System (D-KEFS; Delis et al. 2001) and vocabulary was assessed using the British Picture Vocabulary Scale (BPVS3; Dunn et al. 2009).

Functional MRI was performed to assess language function in 15 of the patients as part of their presurgical workup. Functional MRI studies were performed on a $3 \mathrm{~T}$ Siemens TIM Trio with a gradient echo imaging sequence. A whole-head echo-planar acquisition protocol was used to measure blood oxygen level-dependent (BOLD) changes during the verb generation task. The BOLD sequence was acquired with the following parameters: TR $3000 \mathrm{~ms}$; TE $30 \mathrm{~ms}$; voxels $3 \times 3 \times 3 \mathrm{~mm}$; field of view $192 \mathrm{~mm}$; 44 contiguous $3 \mathrm{~mm}$ thick axial slices. A full volume $1 \mathrm{~mm}^{3}$ high resolution anatomical MRI was acquired using an inversion recovery sequence in the sagittal plane to co-register with the patient's fMRI data.

Data were pre-processed and analysed using Statistical Parametric Mapping software (SPM12; http://www. fil.ion.ucl.ac.uk/spm/). Data for each participant were pre-processed using slice time correction, followed by realignment where the mean image was used as reference. Data were normalised to the standard SPM12 MNI EPI template and a $6 \mathrm{~mm}^{3}$ full width by half maximum (FWHM) isotropic gaussian kernel was used to spatially smooth the normalised images. Statistical analysis at the individual level was estimated using the general linear model and the LI Toolbox (Wilke and Schmithorst 2006) was used to compute language lateralisation indices in the inferior frontal gyrus (Broca's area) and superior temporal gyrus (Wernicke's area). In line with previous studies, patients with indices $>0.2$ were considered left-dominant and patients with indices $<0.2$ were considered to show atypical (right or bilateral) language dominance (Berl et al. 2014).

\section{Direct Cortical Stimulation}

Direct Cortical Stimulation was performed in 10 out of 28 patients according to an established method (Jayakar et al. 1992). Contiguous electrodes pairs were stimulated using $5 \mathrm{~s}$ duration trains of square-wave current pulses of alternating polarity, $0.3 \mathrm{~ms}$ duration, with a frequency of $50 \mathrm{~Hz}$. Stimulus intensity was increased by $1 \mathrm{~mA}$ steps until either one of the following was observed: after-discharge on the intracranial EEG; a clinical response with speech arrest, or an electrographically-documented seizure. 


\section{Results}

All of the patients performed the task successfully, with at least $95 \%$ of the trials correctly completed. This was assessed on the basis of their overt responses to the stimuli. The verb generation task was associated with significant decreases in beta oscillatory power $(13-30 \mathrm{~Hz})$ in frontal and temporal lobes. While the topography of maximum spectral power decrease varied at the voxel level across patients, its location was in the superior temporal gyrus (STG) and inferior frontal gyrus (IFG), typically extending to include areas of the precentral gyrus and premotor cortex. The power decrease generally commenced 250-500 ms after noun presentation and was sustained for approximately $1000 \mathrm{~ms}$. In most patients, the response
A

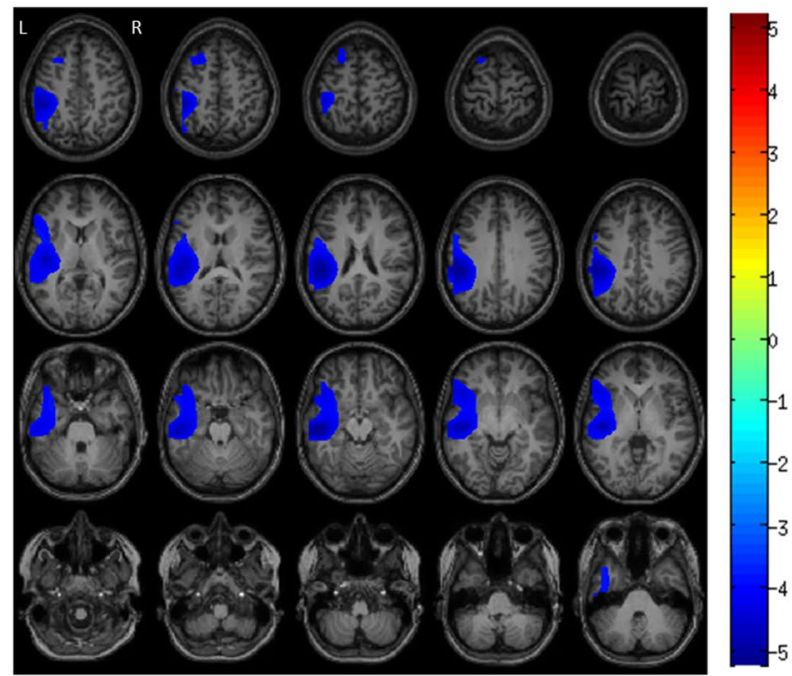

Left Temporal VE

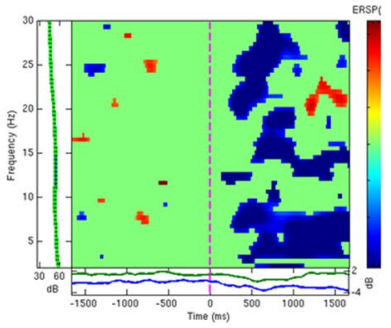

Left Frontal VE

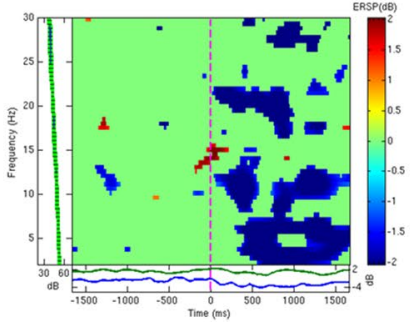

Fig. 1 a Beamformer source localization results of verb generation task for an individual patient (Pt. 13) are shown overlaid on axial slices of the patient's T1 MRI. Sources showing significant decreases in beta oscillatory power $(13-30 \mathrm{~Hz})$ from $2.5 \mathrm{~s}$ pre- to $2.5 \mathrm{~s}$ poststimulus onset were identified in left temporal and left frontal regions $(L I=64.95)$. Time-frequency analysis of a virtual electrode (VE) constructed in the left temporal lobe shows an early sustained decrease in beta power during covert verb generation. Similarly, time-frequency analysis of a virtual electrode constructed in the left frontal lobe shows decreases in beta power from around $200 \mathrm{~ms}$ during covert verb generation. b Top: Electrode grid co-registered and was sustained throughout the whole active phase of the task (e.g. see Fig. 1).

The lateralization of power reduction in the beta frequency band was concordant with language dominance determined by one or more assessment methods (i.e. cortical stimulation mapping, neuropsychological, fMRI or post-operative data) in $89 \%$ of patients (see Table 1). None of the patients who proceeded to surgery displayed any language deficits compared to their pre-surgical status at oneyear follow up. In all twenty-eight cases the MEG data were compared with other available presurgical non-invasive data including neuropsychological and fMRI data on language lateralization where available. Ten right-handed patients had direct cortical stimulation mapping and the results of the MEG analysis was concordant with both the non-invasive and invasive data, confirming left hemisphere lateralization in all ten patients (see Table 1). Figure 1 provides an
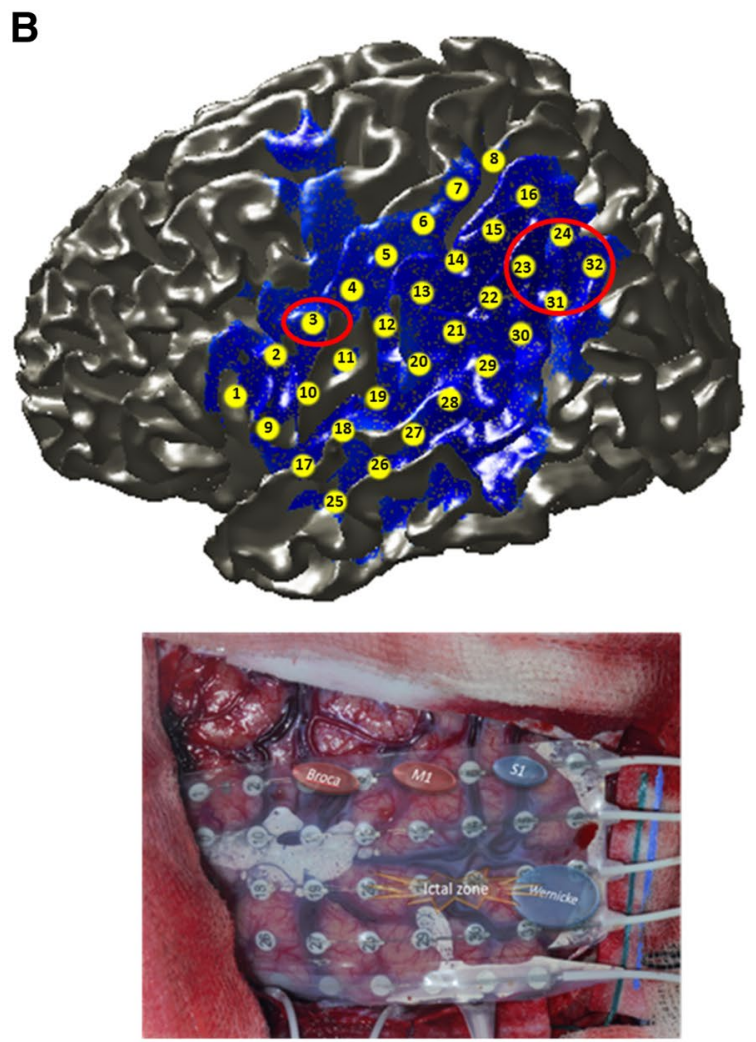

overlaid on cortical surface of the patients MRI along with MEG beamformer source localization results. Red circles indicate location of Wernicke's and Broca's areas as identified during direct cortical stimulation. The peak source of decreased beta power $(13-30 \mathrm{~Hz})$ localized by MEG corresponds to Wernicke's area in this patient. Bottom: Photograph of 32-channel grid shows results from cortical stimulation, where Broca's area was mapped to contact 3 and Wernicke's area to contacts 23-24 and 31-32. When these contacts were stimulated the patient had difficulty producing verbs, was confused and stuttered 
illustrative example of an individual patient (Pt. 13) showing good concordance between the non-invasive MEG language lateralization data and invasive cortical stimulation mapping. MEG sources of decreased beta oscillatory power were identified in left temporal and frontal regions during verb generation and corresponded closely to language regions identified during cortical stimulation mapping (see Fig. 1b). Time-frequency analysis of MEG virtual electrodes constructed in left frontal and temporal regions revealed early sustained responses within $200 \mathrm{~ms}$ of word onset (see Fig. 1a).

In the remaining eighteen cases where cortical stimulation was deemed not clinically appropriate and therefore not performed, MEG results were compared against a combination of neuropsychological, fMRI and post-operative data as clinically available. In this group, MEG data revealed twelve patients with left hemisphere lateralization, four patients with right lateralization, and two patients with bilateral representation. Eleven out of twelve patients showing left hemisphere lateralization with MEG, were confirmed to be left hemisphere dominant based on the available clinical data. Two out of four patients showing right lateralization

\section{A}

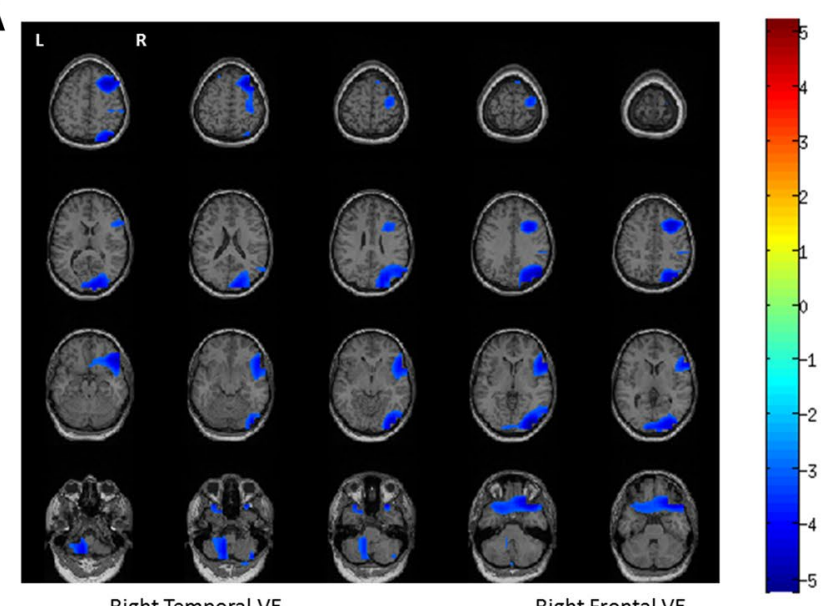

Right Temporal VE

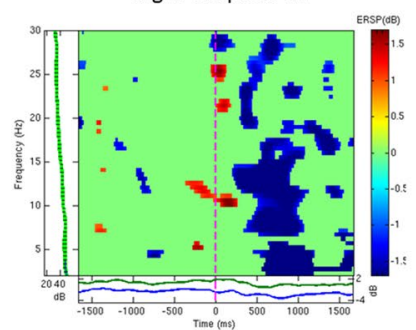

Right Frontal VE

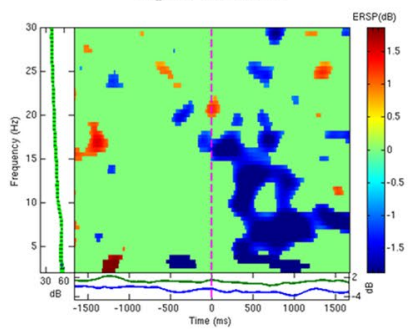

Fig. 2 a Beamformer source localization results of verb generation task for an individual patient (Pt. 11) are shown overlaid on axial slices of the patient's T1 MRI. Sources showing significant decreases in beta oscillatory power $(13-30 \mathrm{~Hz})$ from $2.5 \mathrm{~s}$ pre- to $2.5 \mathrm{~s}$ poststimulus onset were identified in right temporal and right frontal regions $(\mathrm{LI}=-47.91)$. Time-frequency analysis of a virtual electrode (VE) constructed in the right temporal lobe shows significant decreases in beta power from approximately $300 \mathrm{~ms}$ post-stimulus onset. Similarly, time-frequency analysis of a virtual electrode con- with MEG also displayed corresponding right hemisphere dominance in the other clinical data. Figure 2 shows MEG and fMRI data for an individual patient (Pt. 11) who displayed right hemisphere lateralization for language ( $\mathrm{LI}=$ -47.91). MEG sources of decreased beta oscillatory power $(13-30 \mathrm{~Hz})$ were identified in right temporal and frontal regions during verb generation and were consistent with sources of increased activation identified with fMRI during the same task (see Fig. 2b). Time-frequency analysis of MEG virtual electrodes constructed in right frontal and temporal regions revealed early responses within $200 \mathrm{~ms}$ of word onset (see Fig. 2a). The two patients who were identified as having bilateral language representation with MEG were concordant with the other clinical data.

With respect to those cases in which there was divergence between the MEG and other available clinical data, one patient (Pt. 14), who was found to have right lateralized language representation with MEG, was described to have left lateralized language based on pre-operative neuropsychological assessment (see Table 1). This patient had a left anterior temporal lobectomy with hippocampectomy and did
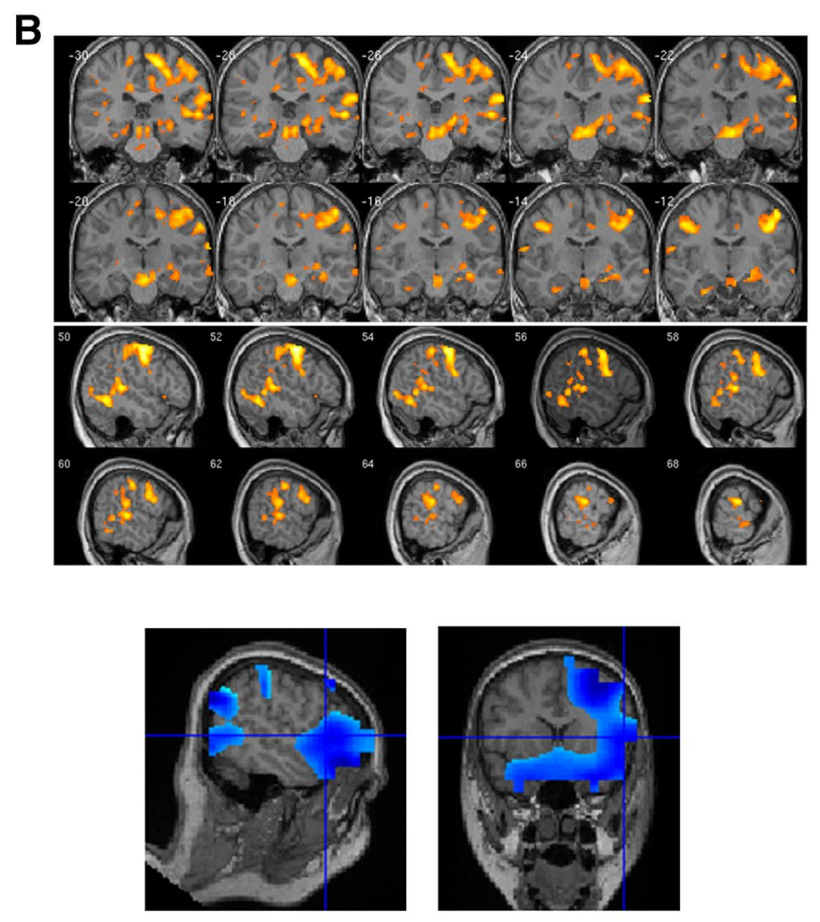

structed in the right frontal lobe shows significant decreases in beta power from approximately $300 \mathrm{~ms}$ post-stimulus onset. b Sources of decreased beta power identified with MEG correspond with fMRI activation during the same covert verb generation task showing right lateralized activation. fMRI activation is shown overlaid on coronal and sagittal slices of the patients MRI. Blue cross-hairs indicate region of peak decrease in MEG beta power in the right inferior frontal gyrus which corresponds to area of increased activation in the fMRI data 
not display any post-operative language deficits at one-year follow-up relative to their pre-surgical competence. Another case (Pt. 17) was found to be right lateralized with MEG but was described as left lateralized based on neuropsychological assessment. This patient had a right frontal lesionectomy and did not display any post-operative language deficits following surgery. In contrast, patient 25 was found to have left lateralized language activity with MEG but fMRI revealed bilateral language activation (see Table 1). Since this patient did not proceed to surgery due to subsequent improvement in seizure frequency, we were unable to further validate the MEG findings.

Overall concordance between MEG lateralization and other clinical data was found in $25 / 28$ patients. This represents $89 \%$ concordance between MEG language lateralization based on beta oscillatory power and a combination of clinical data, including cortical stimulation mapping, fMRI, neuropsychological and post-operative data.

\section{Discussion}

The aim of the present study was to examine feasibility and efficacy of MEG to identify hemispheric dominance for language in a group of pediatric patients in the context of pre-surgical evaluation for drug-resistant focal epilepsy. Our findings have shown that hemispheric dominance for language can be reliably determined with MEG in our sample of children using a verb generation task and beamformerbased spatial filtering. We found significant decreases in beta $(13-30 \mathrm{~Hz})$ oscillatory power primarily in the dominant hemisphere that peaked approximately $500 \mathrm{~ms}$ after stimulus onset. Spectral analysis within pre-defined regions of interest in bilateral inferior frontal and superior temporal regions were used to further elucidate the temporal progression and oscillatory dynamics within the language network during covert verb generation. This provided the opportunity to assess the level of bi-hemispheric oscillatory dynamics within specified regions of the language network during verb generation.

We found an initial decrease in beta power bilaterally in superior temporal regions, followed by significantly greater sustained power decreases in the dominant hemisphere in fronto-temporal regions. In many cases the non-dominant hemisphere showed increases in beta power after around $500 \mathrm{~ms}$, which is consistent with previous findings in adults (Fisher et al. 2008). Previous language studies in adult epilepsy patients (Findlay et al. 2012; Hirata et al. 2010, 2004; Kim and Chung 2008), healthy adult participants (Passaro et al. 2011) and adolescent participants (Pang et al. 2011) using similar beamformer analysis methods have shown robust decreases in beta oscillatory power in fronto-temporal regions in the dominant hemisphere. Notably some of these studies did not focus on the emerging dynamics of hemispheric dominance during language processing as they examined a single time window only or did not explore the virtual electrode outputs (Hirata et al. 2010, 2004; Pang et al. 2011). Potentially failing to detect the early bilateral activation seen in this study and also reported previously in a verb generation task (Findlay et al. 2012) and during a semantic decision-task (McDonald et al. 2010).

Our motivation to focus on induced oscillatory activity rather than concentrating on evoked activity like many previous clinical MEG studies was guided by increasing evidence on the significant role of neural oscillations in binding and information transfer between brain regions involved in cortical processing (Buzsaki and Draguhn 2004; Engel et al. 2001; Roopun et al. 2008). Beta oscillatory activity has been shown to be a clinically relevant measure of hemispheric dominance in a large cohort of adult epilepsy patients (Hirata et al. 2010). In terms of the functional relevance of neural oscillations during language tasks, beta frequency oscillations have been linked to various aspects of language processing including top-down mechanisms (Weiss and Mueller 2012). In this context, we postulate that decreased beta power in the dominant hemisphere may reflect activation and greater involvement of fronto-temporal regions during language processing, whereas the often observed increases in power on the contralateral hemisphere may represent a measure of the interplay between homologous language-relevant regions (Fisher et al. 2008).

This study was designed as a pragmatic retrospective validation study. We assessed the reliability of our MEG language localization method against other available clinical information. In adult studies the IAP has regularly been used as the gold-standard for language lateralization to validate MEG findings (Findlay et al. 2012; Hirata et al. 2010; Papanicolaou et al. 2004). The relationship with pre-surgical electro-cortical stimulation in the context of chronic invasive recordings performed to confirm pre-operative lateralization hypothesis has been reported only sporadically (Simos et al. 1999b). In these patients, good concordance between MEG and invasive language mapping procedures (75-95\%) was seen. However, performing such invasive procedures in pediatric age is not always possible, increasing the difficulty in systematic validation of clinical MEG protocols in this age group. We therefore validated our findings by using a combination of clinical measures that were available, including cortical stimulation mapping, fMRI, neuropsychological and post-operative data where possible. Overall, we found a high level of concordance of $89 \%$ between our MEG language and those established using other clinical measures.

Functional MRI, which is a more mature clinical tool, has recently been used as a non-invasive measure to validate MEG mapped language (for review see Balter et al. 2016). Good levels of concordance between the two modalities have 
been reported when using similar language paradigms (Pang et al. 2011; Wang et al. 2012). There is a growing body of evidence showing that at the neural level, increased BOLD signal is associated with decreased cortical oscillatory power in alpha and beta frequency ranges in MEG (Hall et al. 2014; Zumer et al. 2010). The fact that the brain electro-magnetic signal is a direct measure of neural function makes MEG and EEG ideal non-invasive complementary tools to fMRI particularly when concerns exist of a potential effect of proximity of the anatomical lesion to the activated volume of interest. This issue has been documented in a study on patients with fMRI-critical lesions who showed lower lateralization indices and higher prevalence of discordant language lateralization with IAP test than patients without such lesions (Wellmer et al. 2009). While our study was not designed to address this issue, there are physical grounds to postulate that this should be less of an issue with MEG.

Since in our study invasive data or IAP was only requested based on clinical grounds, we cannot provide systematic validation of our findings that allows comparison with data from other studies in which IAP was performed. However, in light of the evidence supporting the predictive value of presurgical neuropsychological and fMRI data on post-operative language outcome in adults (Binder et al. 2008), we have used them as a useful measure to aid in the validation of MEG language lateralization. In reallife situations, a multimodal approach is still widely used when determining suitability for surgery and our data show that MEG language mapping can provide additional reliable non-invasive information during presurgical evaluation in pediatric epilepsy patients, particularly in younger patients and those with lesions. This is also in line with recent recommendations from the American Clinical MEG Society (Bagic et al. 2017).

We found two cases where our MEG lateralization results did not match the pre-operative neuropsychological assessment data. One patient (Pt. 14) was found to have right lateralized language representation with MEG, but was described to have left lateralized language based on neuropsychological assessment (see Table 1). This patient had a left temporal focal cortical dysplasia and was left handed, factors consistent with having atypical language representation (Berl et al. 2014). Furthermore, this patient proceeded to have a left anterior temporal lobectomy with hippocampectomy and did not display any post-operative language deficits at one year follow-up. Taken together, these factors raise the possibility that this patient had atypical/right language lateralization, consistent with our MEG findings. In cases in which discordant data occur, future studies should include additional sources of information regarding language dominance to better understand the usefulness of MEG in a clinical context.

In another patient (Pt. 17), MEG data showed right hemisphere lateralization but neuropsychological assessment suggested left hemisphere lateralization for language. This patient had tuberous sclerosis complex and an early age of epilepsy onset (age 3). These factors can affect development of the language network and may lead to atypical representation in some cases (Berl et al. 2014). This patient had a frontal lesionectomy to remove a lesion in the right middle frontal gyrus and did not display any post-operative language deficits relative to their pre-surgical status. MEG data showed decreases in beta activity in right inferior frontal and superior temporal lobes, regions outside of the resected area. In the absence of cortical stimulation or fMRI data for this patient it is difficult to draw further conclusions about lateralization in this complex case. While presurgical language and memory assessments in children are useful, they are not highly predictive of postoperative outcome (Lah 2004). Hence the need for a multimodal approach in order to establish language lateralization in children with epilepsy particularly in more complex cases. Notably in our study we found that approximately $21 \%$ of patients demonstrated atypical language lateralization based on our MEG analysis, where $4 / 28$ (14\%) were right lateralized and 2/28 (7\%) showed bilateral language representation. This is consistent with previous work showing that atypical language lateralization occurs in approximately $20-30 \%$ of epilepsy patients (Berl et al. 2014; Springer et al. 1999). We also had a relatively high number of left-handed patients in our cohort $6 / 28(21 \%)$ which may have influenced the results given that left handedness and atypical language representation tend to co-occur in higher frequency than for right-handed individuals. In addition, the age range of patients should also be considered when assessing language lateralization as it has been shown that development is characterized by a shift from bilateral to lateralized activation (Kadis et al. 2011; Ressel et al. 2008). By systematically integrating patientspecific clinical and demographic characteristics with noninvasive neuroimaging data as outlined in the current study, patient-specific profiles can be produced that provide valuable information in the presurgical evaluation process (Balter et al. 2016).

Overall our results revealed broad areas of activation predominantly in frontal (BA 6/44/45/47) and temporal regions (BA 21/22/37) during an expressive verb generation task. Pre-defined regions of interest within frontal (BA 6/44/45) and temporal (BA 22) areas were used to provide an estimate of hemispheric dominance. These specific regions were chosen as they represent areas that are commonly explored with direct cortical stimulation and they are in line with volumes of interest used in previous clinical MEG studies of language in adults (Findlay et al. 2012; Hirata et al. 2004). However, in many surgical cases more precise localization of language areas is required. While this can be inherently difficult due to the complex nature of language processing, it has been shown that the sensitivity and specificity of 
MEG localization can be increased by using a combination of expressive and receptive language tasks (Findlay et al. 2012). Importantly region of interest analysis is a useful tool in this context, particularly the virtual electrode method described here, as it allows more detailed assessment of the dynamic profiles of language processing within specific regions of the language network. Future MEG studies with larger cohorts of paediatric patients using a combination of receptive and expressive language tasks may provide a means of exploring more precise localizations within the language network.

This study has inherent limitations. Due to its pragmatic nature, a comparison of the tolerability of MEG versus fMRI in this cohort of patients was not possible; future studies to benchmark the two techniques against specific outcome measures might offer some insight in establishing clinical indications for each of the modalities. Furthermore, this study focused on the analysis of changes in MEG spectral power, which are used to detect "local" changes in synchronous activity and provide a good measure of hemispheric dominance. This was driven by the consolidated knowledge of the role of beta oscillations in processing semantic and syntactic aspects of language (Weiss and Mueller 2012) but fails to capture the complexity of network interactions within the language network. In conclusion, our findings suggest that MEG-based measures of language lateralization can provide clinically relevant information in assessing patients from early childhood. Our data suggest that qualitative hemispheric differences in task-related changes of spectral power could also offer a promising insight into the contribution of dominant and non-dominant hemispheres in language processing and may help to characterize the specialization and lateralization of language processes in pediatric age.

Funding This study is supported by Birmingham Children's Hospital Charities (BCHRF280)

Open Access This article is distributed under the terms of the Creative Commons Attribution 4.0 International License (http://creativeco mmons.org/licenses/by/4.0/), which permits unrestricted use, distribution, and reproduction in any medium, provided you give appropriate credit to the original author(s) and the source, provide a link to the Creative Commons license, and indicate if changes were made.

\section{References}

Abou-Khalil B (2007) An update on determination of language dominance in screening for epilepsy surgery: the Wada test and newer. noninvasive alternatives Epilepsia 48:442-455. https://doi.org/1 0.1111/j.1528-1167.2007.01012.x

Adjamian P et al (2004) Co-registration of magnetoencephalography with magnetic resonance imaging using bite-bar-based fiducials and surface-matching. Clin Neurophysiol 115:691-698. https:// doi.org/10.1016/j.clinph.2003.10.023

Bagic AI, Bowyer SM, Kirsch HE, Funke ME, Burgess RC, Committee APS (2017) American Clinical MEG Society (ACMEGS) Position Statement \#2: The Value of Magnetoencephalography (MEG)/Magnetic Source Imaging (MSI) in noninvasive presurgical mapping of eloquent cortices of patients preparing for surgical interventions. J Clin Neurophysiol 34:189-195. https://doi. org/10.1097/WNP.0000000000000366

Balter S, Lin G, Leyden KM, Paul BM, McDonald CR (2016) Neuroimaging correlates of language network impairment and reorganization in temporal lobe epilepsy. Brain Lang. https://doi. org/10.1016/j.bandl.2016.06.002

Berl MM et al (2014) Characterization of atypical language activation patterns in focal epilepsy. Ann Neurol 75:33-42. https:// doi.org/10.1002/ana.24015

Binder JR, Sabsevitz DS, Swanson SJ, Hammeke TA, Raghavan M, Mueller WM (2008) Use of preoperative functional MRI to predict verbal memory decline after temporal lobe epilepsy surgery. Epilepsia 49:1377-1394. https://doi.org/10.111 $1 / \mathrm{j} .1528-1167.2008 .01625 . \mathrm{x}$

Bowyer SM et al (2005) Language laterality determined by MEG mapping with MR-FOCUSS. Epilepsy Behav 6:235-241. https ://doi.org/10.1016/j.yebeh.2004.12.002

Breier JI, Simos PG, Wheless JW, Constantinou JE, Baumgartner JE, Venkataraman V, Papanicolaou AC (2001) Language dominance in children as determined by magnetic source imaging and the intracarotid amobarbital procedure: a comparison. J Child Neurol 16:124-130

Buzsaki G, Draguhn A (2004) Neuronal oscillations in cortical networks. Science 304:1926-1929. https://doi.org/10.1126/scien ce. 1099745

Chlebus P, Mikl M, Brazdil M, Pazourkova M, Krupa P, Rektor I (2007) fMRI evaluation of hemispheric language dominance using various methods of laterality index calculation. Exp Brain Res 179:365-374. https://doi.org/10.1007/s00221-006-0794-y

de Ribaupierre S et al (2012) Presurgical language mapping in children with epilepsy: clinical usefulness of functional magnetic resonance imaging for the planning of cortical stimulation. Epilepsia 53:67-78. https://doi.org/10.111 $1 /$ j.1528-1167.2011.03329.x

Delis DC, Kaplan E, Kramer JH (2001) Delis-Kaplan executive function system:technical manual. Harcourt Assessment Company, San Antonio

Delorme A, Makeig S (2004) EEGLAB: an open source toolbox for analysis of single-trial EEG dynamics including independent component analysis. J Neurosci Methods 134:9-21. https://doi. org/10.1016/j.jneumeth.2003.10.009

Demonet JF, Thierry G, Cardebat D (2005) Renewal of the neurophysiology of language: functional neuroimaging. Physiol Rev 85:49-95. https://doi.org/10.1152/physrev.00049.2003

Doss RC, Zhang W, Risse GL, Dickens DL (2009) Lateralizing language with magnetic source imaging: validation based on the Wada test. Epilepsia 50:2242-2248. https://doi.org/10.111 1/j.1528-1167.2009.02242.x

Duchowny M, Jayakar P, Harvey AS, Resnick T, Alvarez L, Dean P, Levin B (1996) Language cortex representation: effects of developmental versus acquired pathology. Ann Neurol 40:31-38. https ://doi.org/10.1002/ana.410400108

Dunn LM, Dunn DM, Styles B, Sewell J (2009) The British Picture Vocabulary Scale III - 3rdEdition. GL Assessment, London

Edwards E, Nagarajan SS, Dalal SS, Canolty RT, Kirsch HE, Barbaro NM, Knight RT (2010) Spatiotemporal imaging of cortical activation during verb generation and picture naming. NeuroImage 50:291-301. https://doi.org/10.1016/j.neuroimage.2009.12.035 
Elliott CD (1990) Differential ability scales. Introductory and technical handbook. The Psychological Corporation, San Antonio

Engel AK, Fries P, Singer W (2001) Dynamic predictions: oscillations and synchrony in top-down processing. Nat Rev Neurosci 2:704-716. https://doi.org/10.1038/35094565

Findlay AM et al (2012) Dynamics of hemispheric dominance for language assessed by magnetoencephalographic imaging. Ann Neurol 71:668-686. https://doi.org/10.1002/ana.23530

Fisher AE et al (2008) Interhemispheric differences of spectral power in expressive language: a MEG study with clinical applications. Int J Psychophysiol 68:111-122. https://doi.org/10.1016/j.ijpsy cho.2007.12.005

Gross J, Kujala J, Hamalainen M, Timmermann L, Schnitzler A, Salmelin R (2001) Dynamic imaging of coherent sources: studying neural interactions in the human brain. Proc Natl Acad Sci U S A 98:694-699. https://doi.org/10.1073/pnas.98.2.694

Hall EL, Robson SE, Morris PG, Brookes MJ (2014) The relationship between MEG and fMRI. NeuroImage $102 \mathrm{Pt}$ 1:80-91. https://doi. org/10.1016/j.neuroimage.2013.11.005

Hamberger MJ, Cole J (2011) Language organization and reorganization in epilepsy. Neuropsychol Rev 21:240-251. https://doi. org/10.1007/s11065-011-9180-z

Hillebrand A, Singh KD, Holliday IE, Furlong PL, Barnes GR (2005) A new approach to neuroimaging with magnetoencephalography. Hum Brain Map 25:199-211. https://doi.org/10.1002/hbm.20102

Hirata $\mathrm{M}$ et al (2004) Determination of language dominance with synthetic aperture magnetometry: comparison with the Wada test. NeuroImage 23:46-53. https://doi.org/10.1016/j.neuroimage .2004 .05 .009

Hirata $M$ et al (2010) Language dominance and mapping based on neuromagnetic oscillatory changes: comparison with invasive procedures. J Neurosurg 112:528-538. https://doi. org/10.3171/2009.7.JNS09239

Holmes GL (2016) Effect of seizures on the developing brain and cognition. Semin Pediatr Neurol 23:120-126. https://doi. org/10.1016/j.spen.2016.05.001

Jansen A et al (2006) The assessment of hemispheric lateralization in functional MRI-robustness and reproducibility. NeuroImage 33:204-217. https://doi.org/10.1016/j.neuroimage.2006.06.019

Jayakar P, Alvarez LA, Duchowny MS, Resnick TJ (1992) A safe and effective paradigm to functionally map the cortex in childhood. J Clin Neurophysiol 9:288-293

Kadis DS, Pang EW, Mills T, Taylor MJ, McAndrews MP, Smith ML (2011) Characterizing the normal developmental trajectory of expressive language lateralization using magnetoencephalography. J Int Neuropsychol Soc 17:896-904. https://doi.org/10.1017/ S1355617711000932

Kim JS, Chung CK (2008) Language lateralization using MEG beta frequency desynchronization during auditory oddball stimulation with one-syllable words. NeuroImage 42:1499-1507. https://doi. org/10.1016/j.neuroimage.2008.06.001

Lah S (2004) Neuropsychological outcome following focal cortical removal for intractable epilepsy in children. Epilepsy Behav $5: 804-817$

Maestu F, Ortiz T, Fernandez A, Amo C, Martin P, Fernandez S, Sola RG (2002) Spanish language mapping using MEG: a validation study. NeuroImage 17:1579-1586

Makeig S (1993) Auditory event-related dynamics of the EEG spectrum and effects of exposure to tones. Electroencephalogr Clin Neurophysiol 86:283-293

Maris E, Oostenveld R (2007) Nonparametric statistical testing of EEG- and MEG-data. J Neurosci Methods 164:177-190. https:// doi.org/10.1016/j.jneumeth.2007.03.024

McDonald CR et al (2010) Multimodal imaging of repetition priming: Using fMRI, MEG, and intracranial EEG to reveal spatiotemporal profiles of word processing. NeuroImage 53:707-717. https://doi. org/10.1016/j.neuroimage.2010.06.069

Merrifield WS, Simos PG, Papanicolaou AC, Philpott LM, Sutherling WW (2007) Hemispheric language dominance in magnetoencephalography: sensitivity, specificity, and data reduction techniques. Epilepsy Behav 10:120-128. https://doi.org/10.1016/j. yebeh.2006.10.012

Nolte G (2003) The magnetic lead field theorem in the quasi-static approximation and its use for magnetoencephalography forward calculation in realistic volume conductors. Phys Med Biol 48:3637-3652

Ojemann S, Berger M, Lettich E et al (2003) Localization of language function in children: results of electrical stimulation mapping. J Neurosurg 98:465-470

Oostenveld R, Fries P, Maris E, Schoffelen JM (2011) FieldTrip: Open source software for advanced analysis of MEG, EEG, and invasive electrophysiological data. Comput Intell Neurosci 2011:156869. https://doi.org/10.1155/2011/156869

Pang EW, Wang F, Malone M, Kadis DS, Donner EJ (2011) Localization of Broca's area using verb generation tasks in the MEG: validation against fMRI. Neurosci Lett 490:215-219. https:// doi.org/10.1016/j.neulet.2010.12.055

Papanicolaou AC et al (2004) Magnetocephalography: a noninvasive alternative to the Wada procedure. J Neurosurg 100:867-876. https://doi.org/10.3171/jns.2004.100.5.0867

Passaro AD, Rezaie R, Moser DC, Li Z, Dias N, Papanicolaou AC (2011) Optimizing estimation of hemispheric dominance for language using magnetic source imaging. Brain Res 1416:4450. https://doi.org/10.1016/j.brainres.2011.08.017

Pirmoradi M, Beland R, Nguyen DK, Bacon BA, Lassonde M (2010) Language tasks used for the presurgical assessment of epileptic patients with MEG. Epilept Disord 12:97-108. https://doi. org/10.1684/epd.2010.0314

Ressel V, Wilke M, Lidzba K, Lutzenberger W, Krageloh-Mann I (2008) Increases in language lateralization in normal children as observed using magnetoencephalography. Brain Lang 106:167176. https://doi.org/10.1016/j.bandl.2008.01.004

Rezaie R, Narayana S, Schiller K, Birg L, Wheless JW, Boop FA, Papanicolaou AC (2014) Assessment of hemispheric dominance for receptive language in pediatric patients under sedation using magnetoencephalography. Front Hum Neurosci 8:657. https:// doi.org/10.3389/fnhum.2014.00657

Rodin D, Bar-Yosef O, Smith ML, Kerr E, Morris D, Donner EJ (2013) Language dominance in children with epilepsy: concordance of fMRI with intracarotid amytal testing and cortical stimulation. Epilepsy Behav 29:7-12. https://doi.org/10.1016/j. yebeh.2013.06.005

Roopun AK et al (2008) Temporal interactions between cortical rhythms. Front Neurosci 2:145-154. https://doi.org/10.3389/ neuro.01.034.2008

Rutten GJ, Ramsey NF, van Rijen PC, Alpherts WC, van Veelen CW (2002) FMRI-determined language lateralization in patients with unilateral or mixed language dominance according to the Wada test. NeuroImage 17:447-460

Simos PG et al (1999a) Atypical temporal lobe language representation: MEG and intraoperative stimulation mapping correlation. Neuroreport 10:139-142

Simos PG, Papanicolaou AC, Breier JI, Wheless JW, Constantinou JE, Gormley WB, Maggio WW (1999b) Localization of language-specific cortex by using magnetic source imaging and electrical stimulation mapping. J Neurosurg 91:787-796. https ://doi.org/10.3171/jns.1999.91.5.0787

Singh KD, Barnes GR, Hillebrand A (2003) Group imaging of taskrelated changes in cortical synchronisation using nonparametric permutation testing. NeuroImage 19:1589-1601 
Springer JA et al (1999) Language dominance in neurologically normal and epilepsy subjects: a functional MRI study. Brain J Neurol 122(Pt 11):2033-2046

Tanaka $\mathrm{N}$ et al (2013) Language lateralization represented by spatiotemporal mapping of magnetoencephalography. Am J Neuroradiol 34:558-563. https://doi.org/10.3174/ajnr.A3233

Taulu S, Hari R (2009) Removal of magnetoencephalographic artifacts with temporal signal-space separation: demonstration with single-trial auditory-evoked responses. Hum Brain Map 30:1524-1534. https://doi.org/10.1002/hbm.20627

Van Poppel M et al (2012) Passive language mapping with magnetoencephalography in pediatric patients with epilepsy. J Neurosurg Pediatr 10:96-102. https://doi.org/10.3171/2012.4.PEDS11301

Wang Y, Holland SK, Vannest J (2012) Concordance of MEG and fMRI patterns in adolescents during verb generation. Brain Res 1447:79-90. https://doi.org/10.1016/j.brainres.2012.02.001

Wechsler D (1999) Wechsler abbreviated scale of intelligence. The psychological corporation. Harcourt Brace \& Company, New York

Weiss S, Mueller HM (2012) "Too many betas do not spoil the broth": the role of beta brain oscillations in language processing. Front Psychol 3:201. https://doi.org/10.3389/fpsyg.2012.00201
Wellmer J, Weber B, Urbach H, Reul J, Fernandez G, Elger CE (2009) Cerebral lesions can impair fMRI-based language lateralization. Epilepsia 50:2213-2224. https://doi.org/10.111 $1 /$ j.1528-1167.2009.02102

Wilke M, Schmithorst VJ (2006) A combined bootstrap = histogram analy- sis approach for computing a lateralization index from neuroimag- ing data. Neuroimage 33:522-530

Zumer JM, Brookes MJ, Stevenson CM, Francis ST, Morris PG (2010) Relating BOLD fMRI and neural oscillations through convolution and optimal linear weighting. NeuroImage 49:1479-1489. https:// doi.org/10.1016/j.neuroimage.2009.09.020

Publisher's Note Springer Nature remains neutral with regard to jurisdictional claims in published maps and institutional affiliations. 